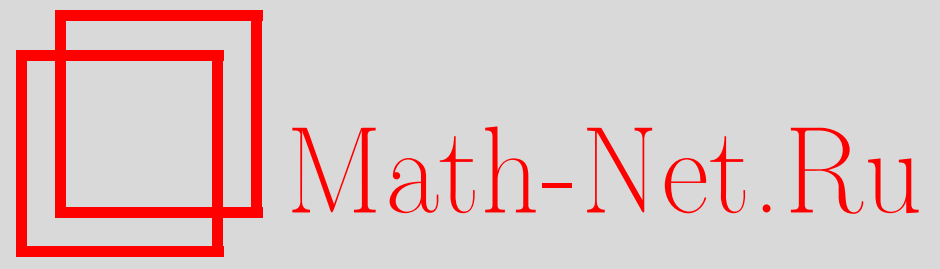

Д. Е. Ефимов, Оценка экспонента некоторых множеств неотрицательных матриц, Дискрет. матем., 1999, том 11, выпуск 4, 79-88

DOI: https://doi.org/10.4213/dm394

Использование Общероссийского математического портала Math-Net.Ru подразумевает, что вы прочитали и согласны с пользовательским соглашением http://www.mathnet.ru/rus/agreement

Параметры загрузки:

IP : 3.89 .197 .203

26 апреля 2023 г., $17: 50: 33$

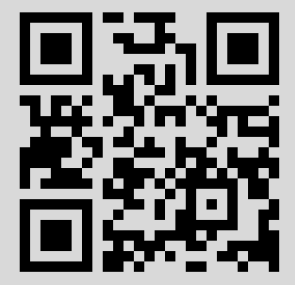




\title{
Оценка экспонента некоторых множеств неотрицательных матриц
}

\author{
(C) 1999 г. Д. Е. Ефимов
}

\begin{abstract}
Экспонентом множества $\mathscr{A}$ неотрицательных $k \times k$ матриц называется такое минимальное $n$, что для любой выборки с возвращением $A_{1}, \ldots, A_{n} \in \mathscr{A}$ все элементы матрицы $A_{1} \ldots A_{n}$ положительны. В статье получены верхние оценки экспонента некоторых множеств матриц с использованием сингулярных чисел матриц. Найдена также оценка экспонента множества матриц, полученных с помощью операции обобщенного кронекерова произведения матриц. Эти результаты используются для оценки длины покрытия группы в заданной системе образующих.
\end{abstract}

\section{1. Введение}

Квадратная неотрицательная матрица $A$ называется примитивной, если существует такое $n \in \mathrm{N}$, что $A^{n}>0$, то есть все элементы матрицы $A^{n}$ положительны. Экспонентом примитивной матрицы $A$ называется величина

$$
\gamma(A)=\min \left\{n \in \mathbf{N}: A^{n}>0\right\} .
$$

Следуя [1], множество $\mathscr{A}$ неотрицательных $k \times k$ матриц будем называть классно примитивным, если существует такое $n \in \mathrm{N}$, что для любой выборки с возвращением $A_{1}, A_{2}, \ldots, A_{n} \in \mathscr{A}$ имеет место неравенство $A_{1} A_{2} \ldots A_{n}>0$. Минимальное из таких чисел $n$ назовем экспонентом множества $\mathscr{A}$ и обозначим через $\gamma(\mathscr{A})$.

В [1] вводится понятие индекса неразложимости и доказывается следующий результат для множества $\mathfrak{A}=\left\{A_{1}, \ldots, A_{n}\right\}$ неотрицательных матриц: если индекс неразложимости каждой матрицы $A_{i}, i=1, \ldots, N$, не меньше некоторого фиксированного числа $r$, где $r \geqslant 1$, то для экспонента этого множества справедлива оценка

$$
\gamma(\mathfrak{A}) \leqslant \begin{cases}(k-1) / r, & \text { если } r \text { делит } k-1, \\ {[(k-1) / r]+1,} & \text { если } r \text { не делит } k-1 .\end{cases}
$$

Во втором параграфе получены верхние оценки экспонента некоторых множеств матриц с использованием теории сингулярных чисел матриц. В третьем параграфе обобщено понятие кронекерова произведения матриц и доказана оценка экспонента множества матриц, полученных при помощи этой операции. Эти результаты используются для получения оценки длины покрытия группы в системе образующих. 


\section{2. Оценка экспонента множества матриц}

Обозначим через $A^{*}$ матрицу, сопряженную матрице $A$. Всюду ниже сингулярные числа матрицы $A$ (неотрицательные квадратные корни собственных чисел матрицы $\left.A^{*} A\right) \sigma_{1}(A), \ldots, \sigma_{k}(A)$ занумерованы в порядке невозрастания их величин (см. [2]).

Пусть $\mathbf{C}(k)$ - множество $k \times k$ матриц над полем комплексных чисел,

$$
r(n)=\operatorname{rang}\left(A_{1}-L\right)\left(A_{2}-L\right) \ldots\left(A_{n}-L\right)
$$

и

$$
\varepsilon(A, B)=\left(\sum_{i=1}^{k} \sum_{j=1}^{k}\left|a_{i j}-b_{i j}\right|^{2}\right)^{1 / 2}
$$

- евклидово расстояние между матрицами.

Теорема 1. Пусть $A_{1}, A_{2}, \ldots, A_{n}, \ldots$ - последовательность матрич из $\mathbf{C}(k)$,

$$
B_{n}=\prod_{t=1}^{n} A_{t}
$$

и при любом $n \in \mathbf{N}$ существует предел

$$
\lim _{t \rightarrow \infty} A_{n}^{t}=L
$$

не зависящий от $n$. Пусть тажже $\sigma_{j}\left(A_{n}-L\right) \leqslant \sigma_{j}, \sigma_{1} \geqslant \sigma_{2} \geqslant \ldots \geqslant \sigma_{k} u \sigma_{j}<1$ при любых $n \in \mathbf{N}, j=1, \ldots, k$. Тогда

$$
\lim _{n \rightarrow \infty} B_{n}=L, \quad \varepsilon\left(L, B_{n}\right) \leqslant\left(\sum_{j=1}^{r(n)} \sigma_{j}^{2 n}\right)^{1 / 2} .
$$

Для доказательства потребуются следующие леммы.

Лемма 1. Пусть $\sigma_{j}\left(A_{i}\right) \leqslant \sigma_{j}$ при любом $j=1, \ldots, k$ и любом $i=1, \ldots, l$. Обозначим через $\sigma_{l, 1}, \ldots, \sigma_{l, k}-$ сингулярные числа матричы

$$
B_{l}=\prod_{i=1}^{l} A_{i}
$$

причем $\sigma_{l, 1} \geqslant \sigma_{l, 2} \geqslant \ldots \geqslant \sigma_{l, k}$.

Тогда

$$
\sum_{j=1}^{m} \sigma_{l, j}^{u} \leqslant \sum_{j=1}^{m} \sigma_{j}^{l u}
$$

для любого $m=1, \ldots, k$ и любого действительного $u \geqslant 0$.

Доказательство повторяет доказательство аналогичного неравенства для последовательности степеней линейного оператора (см. [3], стр. 508).

Лемма 2. Пусть $L^{2}=A_{i} L=L A_{i}=L$ для любого $i \in \mathrm{N}$. Тогда

$$
\prod_{t=1}^{n} A_{t}-L=\prod_{t=1}^{n}\left(A_{t}-L\right)
$$


Доказательство. Доказательство проведем индукцией по $n$.

При $n=1$ равенство (1) очевидно. Предположим, что равенство (1) верно для $n-1$, то есть

$$
\prod_{t=1}^{n-1} A_{t}-L=\prod_{t=1}^{n-1}\left(A_{t}-L\right)
$$

Докажем равенство для $n$. Нетрудно видеть, что

$$
\begin{aligned}
\prod_{t=1}^{n}\left(A_{t}-L\right) & =\left(\prod_{t=1}^{n-1}\left(A_{t}-L\right)\right)\left(A_{n}-L\right)=\left(\prod_{t=1}^{n-1} A_{t}-L\right)\left(A_{n}-L\right) \\
& =\prod_{t=1}^{n} A_{t}-L A_{n}-\prod_{t=1}^{n-1} A_{t} L+L^{2}=\prod_{t=1}^{n} A_{t}-\prod_{t=1}^{n-1} A_{t} L \\
& =\prod_{t=1}^{n} A_{t}-\prod_{t=1}^{n-2} A_{t} L=\ldots=\prod_{t=1}^{n} A_{t}-L
\end{aligned}
$$

Лемма 3. Пусть предел $A^{t}$ прu $t \rightarrow \infty$ существует и равен L. Тогда

$$
A L=L A=L^{2}=L .
$$

Доказателъство. Ясно, что

$$
A L=A \lim _{t \rightarrow \infty} A^{t}=\lim _{t \rightarrow \infty} A^{t+1}=L .
$$

Другие равенства доказываются аналогично.

Будем обозначать $\operatorname{tr} A$ след матрицы $A$.

Доказательство теоремъ 1. Пусть $\sigma_{n, 1} \geqslant \sigma_{n, 2} \geqslant \ldots \geqslant \sigma_{n, k}-$ сингулярные числа матрицы $\prod_{t=1}^{n}\left(A_{t}-L\right)$, упорядоченные по невозрастанию. Из [4] (см. упражнение 1 , стр. 196 в [4]) следует, что

$$
\varepsilon\left(B_{n}, L\right)=\sqrt{\operatorname{tr}\left(B_{n}-L\right)\left(B_{n}-L\right)^{*}} .
$$

Из лемм 2 и 3 следует, что

$$
B_{n}-L=\prod_{t=1}^{n}\left(A_{t}-L\right)
$$

Отсюда,

$$
\varepsilon\left(B_{n}, L\right)=\left(\operatorname{tr} \prod_{t=1}^{n}\left(A_{t}-L\right)\left(\prod_{t=1}^{n}\left(A_{t}-L\right)\right)^{*}\right)^{1 / 2}=\left(\sum_{j=1}^{k} \sigma_{n, j}^{2}\right)^{1 / 2}
$$

Количество ненулевых сингулярных чисел равно рангу:

$$
\operatorname{rang}\left(B_{n}-L\right)=\operatorname{rang}\left(\prod_{t=1}^{n}\left(A_{t}-L\right)\right)=r(n) .
$$


Поэтому

$$
\varepsilon\left(B_{n}, L\right)=\left(\sum_{j=1}^{r(n)} \sigma_{n, j}^{2}\right)^{1 / 2}
$$

Из леммы 1 при $m=r(n)$ и $u=2$ следует, что

$$
\varepsilon^{2}\left(B_{n}, L\right)=\sum_{j=1}^{r(n)} \sigma_{n, j}^{2} \leqslant \sum_{j=1}^{r(n)} \sigma_{j}^{2 n} .
$$

По условию теоремы $\sigma_{j}<1$ при любом $j=1, \ldots, k$, поэтому

$$
\lim _{n \rightarrow \infty} \varepsilon\left(B_{n}, L\right)=0 \text {. }
$$

Следовательно,

$$
\lim _{n \rightarrow \infty} B_{n}=L \text {. }
$$

Лемма 4. Существует унитарная $k \times k$ матрица $U$, приводящая любую дваждъ стохастическую $k \times k$ матричу $P \propto$ вполне распавшемуся виду, то есть

$$
U^{*} P U=\left(\begin{array}{cc}
1 & \overrightarrow{0}_{k-1} \\
0_{k-1}^{\downarrow} & T(P)
\end{array}\right),
$$

где $T(P)$ - некоторая $(k-1) \times(k-1)$ матрица, а $\overrightarrow{0}_{k-1} u 0_{k-1}^{\downarrow}-$ строка и столбец из $k-1$ нулей.

Доказательство проводится прямым построением искомой матрицы $U$.

Обозначим через

$$
\|\vec{a}\|=\left(\sum_{i=1}^{k}\left|a_{i}\right|\right)^{1 / 2}
$$

норму вектора-строки $\vec{a}=\left(a_{1}, \ldots, a_{k}\right)$, пусть также $\vec{a}_{i}-i$-я строка, $a_{i}^{\downarrow}-i$-й столбец матрицы $A$.

Воспользовавшись неравенством Коши-Буняковского-Шварца, можно доказать следующую лемму.

Лемма 5. Пустъ $C \in \mathbf{C}(k)$ и $A=B C D$, максимальное значение нормы строки матрицы $B$ равно $b$, а столбца матрицы $D$ равно $d$. Тогда

$$
\max \left\{\left|a_{i j}\right| \mid i, j=1, \ldots, k\right\} \leqslant b d \sigma_{1}(C) .
$$

Теорема 2. Пусть $\mathscr{A}-$ такое множество неотрицательных $k \times k$ матрич, что для любой $A \in \mathscr{A}$ существует $\lim _{t \rightarrow \infty} A^{t}=L u \sigma_{1}(A-L) \leqslant \sigma_{1}<1$. Пусть $L-$ положительная матрица, $l-е е$ минимальный элемент $u$ $b=\sup \left\{\left\|\vec{a}_{i}-\vec{l}_{i}\right\| \mid A \in \mathscr{A}, i=1, \ldots, k\right\}, \quad d=\sup \left\{\left\|a_{j}^{\downarrow}-l_{j}^{\downarrow}\right\| \mid A \in \mathscr{A}, j=1, \ldots, k\right\}$. Тогда $\mathscr{A}$ классно примитивно и

$$
\gamma(\mathscr{A}) \leqslant \begin{cases}1, & \text { если } \sigma_{1}=0, \\ 2, & \text { если } \sigma_{1}>0, \quad b d<l \\ \left\lfloor(\ln l-\ln b-\ln d) / \ln \sigma_{1}\right\rfloor+3, & \text { если } \sigma_{1}>0, \quad b d \geqslant l .\end{cases}
$$


Доказательство. Пусть $\sigma_{1}=0$. Тогда $\varepsilon(A, L)=0$ и $A=L>0$ при любой $A \in \mathscr{A}$. Значит, $\gamma(\mathscr{A})=1$.

Докажем оценку для случая $b d<l$ от противного. Если существуют $A_{1}, A_{2}$ из $\mathscr{A}$ такие, что $A_{1} A_{2}$ содержит нулевые элементы, то $A_{1} A_{2}-L$ содержит элемент, больший, либо равный по модулю $l$. Согласно лемме 5 это невозможно.

Докажем оценку для случая $\sigma_{1}>0$ и $b d \geqslant l$ от противного.

Пусть для $n \geqslant\left\lfloor(\ln l-\ln b-\ln d) / \ln \sigma_{1}\right\rfloor+3$ существует набор $A_{1}, \ldots, A_{n} \in \mathscr{A}$ такой, что $c_{r s}=0$, где $A_{1} A_{2} \ldots A_{n}=\left(c_{i j}\right)=C$. Тогда при $B=\left(b_{i j}\right)=C-L$ справедливо неравенство

$$
l \leqslant\left|-l_{r s}\right|=\left|c_{r s}-l_{r s}\right|=\left|b_{r s}\right| .
$$

По лемме 5

$$
\left|c_{r s}-l_{r s}\right| \leqslant b d \sigma_{1}\left(\left(A_{2}-L\right) \ldots\left(A_{n-1}-L\right)\right) .
$$

По теореме Неймана-Хорна (см. [3], стр.507) для сингулярных чисел имеют место неравенства

$$
\sigma_{1}\left(\prod_{j=2}^{n-1}\left(A_{j}-L\right)\right) \leqslant \prod_{j=2}^{n-1} \sigma_{1}\left(A_{j}-L\right) \leqslant \sigma_{1}^{n-2} .
$$

По определению логарифма $\sigma_{1}=\exp \left(\ln \sigma_{1}\right)$. Подставив это выражение в неравенство (4), получаем, что

$$
\left|c_{r s}-l_{r s}\right| \leqslant b d \sigma_{1}\left(\left(A_{2}-L\right) \ldots\left(A_{n-1}-L\right)\right) \leqslant b d \exp \left((n-2) \ln \sigma_{1}\right) .
$$

Поскольку $\ln \sigma_{1}<0, n \geqslant\left\lfloor(\ln l-\ln b-\ln d) / \ln \sigma_{1}\right\rfloor+3$ и экспонента - строго возрастающая функция, справедлива оценка

$$
\left|c_{r s}-l_{r s}\right|<b d \exp \left(\left(\frac{\ln l-\ln b-\ln d}{\ln \sigma_{1}}+2-2\right) \ln \sigma_{1}\right) .
$$

После арифметических преобразований в правой части неравенства получаем, что

$$
\left|c_{r s}-l_{r s}\right|<b d \exp (\ln l-\ln b-\ln d)=b d \frac{l}{b d}=l .
$$

Сопоставив это неравенство с неравенством (3), получаем противоречие.

Теорема 3. Пусть $\mathscr{A}$ - множество дважды стохастических матрич размера $k \times k u \sigma_{2}(A) \leqslant \sigma_{2}<1$ для любой $A \in \mathscr{A}, L$ - матрича размера $k \times k$ с элементами $1 / k$,

$$
b=\sup \left\{\left\|\vec{a}_{i}-\vec{l}_{i}\right\| \mid A \in \mathscr{A}, i=1, \ldots, k\right\}, \quad d=\sup \left\{\left\|a_{j}^{\downarrow}-l_{j}^{\downarrow}\right\| \mid A \in \mathscr{A}, j=1, \ldots, k\right\} .
$$

Тогда множество $\mathscr{A}$ классно примитивно и

$$
\gamma(\mathscr{A}) \leqslant \begin{cases}1, & \text { если } \sigma_{2}=0, \\ 2, & \text { если } \sigma_{2}>0, b d<k^{-1}, \\ \left\lfloor(\ln k+\ln b+\ln d) / \ln \sigma_{2}^{-1}\right\rfloor+3, & \text { если } \sigma_{2}>0, \text { bd } \geqslant k^{-1}\end{cases}
$$


Доказательство. Докажем, что множество $\mathscr{A}$ удовлетворяет условию теоремы 2. Из дважды стохастичности следует, что $A L=L A=A$ для любой $A \in \mathscr{A}$.

Как уже отмечалось,

$$
\varepsilon\left(A^{t}, L\right)=\sqrt{\operatorname{tr}\left(A^{t}-L\right)\left(A^{t}-L\right)^{*}} .
$$

Отсюда, раскрыв скобки в правой части равенства, получаем, что

$$
\varepsilon\left(A^{t}, L\right)=\sqrt{\operatorname{tr}\left(A^{t} A^{* t}\right)-\operatorname{tr} L} .
$$

Учитывая неравенство для следов матриц $\operatorname{tr}\left(A^{* t} A^{t}\right) \leqslant \operatorname{tr}\left(A^{*} A\right)^{t}$ (см. [3], стр. 508), получаем равенство

$$
\varepsilon\left(A^{t}, L\right)=\sqrt{\operatorname{tr}\left(A^{t} A^{* t}\right)-1} .
$$

Из определения сингулярных чисел следует, что

$$
\varepsilon\left(A^{t}, L\right)=\left(\sum_{j=1}^{k} \sigma_{j}^{2 t}(A)-1\right)^{1 / 2}
$$

Поскольку все матрицы из $\mathscr{A}$ дважды стохастические, первое сингулярное число каждой матрицы равно 1. Отсюда,

$$
\varepsilon\left(A^{t}, L\right)=\left(1+\sum_{j=2}^{k} \sigma_{j}^{2 t}(A)-1\right)^{1 / 2}=\left(\sum_{j=2}^{k} \sigma_{j}^{2 t}(A)\right)^{1 / 2} \leqslant \sigma_{2}^{t}(A) \sqrt{k-1} \leqslant \sigma_{2}^{t} \sqrt{k-1}
$$

По условию теоремы $\sigma_{2}<1$. Поэтому

$$
\lim _{t \rightarrow \infty} \varepsilon\left(A^{t}, L\right)=0
$$

и по определению

$$
\lim _{t \rightarrow \infty} A^{t}=L
$$

для любой $A \in \mathscr{A}$.

Найдем $\sigma_{1}(A-L)$. Поскольку умножение на унитарную матрицу не меняет сингулярные числа, для $U$ согласно лемме 4 верна цепочка равенств

$$
\begin{aligned}
\sigma_{1}(A-L) & =\sigma_{1}\left(U^{*}(A-L) U\right)=\sigma_{1}\left(U^{*} A U-U^{*} L U\right) \\
& =\sigma_{1}\left(\left(\begin{array}{cc}
1 & \overrightarrow{0}_{k-1} \\
0_{k-1}^{\downarrow} & T(A)
\end{array}\right)-\left(\begin{array}{cc}
1 & \overrightarrow{0}_{k-1} \\
0_{k-1}^{\downarrow} & 0_{(k-1) \times(k-1)}
\end{array}\right)\right)=\sigma_{2}(A),
\end{aligned}
$$

где $T(A)$ - матрица размера $(k-1) \times(k-1)$, определенная в лемме 4 , а $0_{(k-1) \times(k-1)}$ - матрица размера $(k-1) \times(k-1)$, состоящая из нулей. Получаем ограничение сверху $\sigma_{1}(A-L) \leqslant \sigma_{2}<1$.

Значит множество матриц $\mathscr{A}$ удовлетворяет условию теоремы 2 . Подставив в формулу (2) значения

$$
\min \left\{l_{i j}: i, j=1, \ldots, k\right\}=1 / k, \quad \sigma_{1}=\sigma_{2}
$$


и поменяв знаки числителя и знаменателя, получим, что

$$
\gamma(\mathscr{A}) \leqslant \begin{cases}1, & \text { если } \sigma_{2}=0 \\ 2, & \text { если } \sigma_{2}>0, b d<k^{-1} \\ \left\lfloor(\ln k+\ln b+\ln d) / \ln \sigma_{2}^{-1}\right\rfloor+3, & \text { если } \sigma_{2}>0, b d \geqslant k^{-1} .\end{cases}
$$

Пусть $A-$ неотрицательная матрица, $\mathscr{Q}(A)-$ множество дважды стохастических матриц $Q=\left\|q_{i j}\right\|$ размера $k \times k$ со свойством $q_{i j}=0$, если $a_{i j}=0$. Пусть $\mathscr{A}-$ множество неотрицательных матриц. Тогда множество $\{\mathscr{Q}(A): A \in \mathscr{A}\}$ конечно.

Обозначим $L$ матрицу размера $k \times k$ с элементами $1 / k$.

Теорема 4. Пусть $\mathscr{A}-$ множество неотрицательных матрич размера $k \times k u$ $\mathscr{Q}(A) \neq \varnothing$ для любой $A \in \mathscr{A}$. Пусть существует такое $\sigma_{2}<1$, ито для любой $A \in \mathscr{A}$ найдется $Q_{0}(A) \in \mathscr{Q}(A)$ такал, что $\sigma_{2}\left(Q_{0}(A)\right) \leqslant \sigma_{2}, \vec{b}_{i}(a)-i$-я строка матрицы $Q_{0}(A)-L$,

$$
b=\sup \left\{\left\|\vec{b}_{i}(A)\right\| \mid A \in \mathscr{A}, i=1, \ldots, k\right\}
$$

$d_{j}^{\downarrow}(A)-j$-й столбец, матрицъ $Q_{0}(A)-L$,

$$
d=\sup \left\{\left\|d_{j}^{\downarrow}(A)\right\| \mid A \in \mathscr{A}, j=1, \ldots, k\right\} .
$$

Tогдa

$$
\gamma(\mathscr{A}) \leqslant \begin{cases}1, & \text { если } \sigma_{2}=0 \\ 2, & \text { если } \sigma_{2}>0, \quad b d<k^{-1} \\ \left\lfloor(\ln k+\ln b+\ln d) / \ln \sigma_{2}^{-1}\right\rfloor+3, & \text { если } \sigma_{2}>0, \text { bd } \geqslant k^{-1 .}\end{cases}
$$

Доказательство. Очевидно, что из неравенства $Q_{0}\left(A_{1}\right) Q_{0}\left(A_{2}\right) \ldots Q_{0}\left(A_{n}\right)>0$ следует, что $A_{1} A_{2} \ldots A_{n}>0$. Теперь оценка следует из теоремы 3 .

Определение 1. Назовем неотрицательную матрицу $m$-регулярной, если в каждой строке и в каждом столбце она содержит ровно $m$ ненулевых элементов.

Для $m$-регулярной матрицы $A$ в случае $m>0$ введем матричную функцию $G(A)$, полагая

$$
g_{i j}= \begin{cases}1 / m, & b_{i j}>0 \\ 0, & b_{i j}=0\end{cases}
$$

Следствие. Пусть $\mathscr{A}-$ множество $m$-регулярных $k \times k$ матриц, $m>0$, $\sigma_{2}(G(A)) \leqslant \sigma_{2}<1$ при любой $A \in \mathscr{A}$. Тогда

$$
\gamma(\mathscr{A}) \leqslant \begin{cases}1, & \text { если } \sigma_{2}=0 \\ 2, & \text { если } \sigma_{2}>0, m>k / 2 \\ \left\lfloor(\ln (k-m)-\ln m) / \ln \sigma_{2}^{-1}\right\rfloor+3, & \text { если } \sigma_{2}>0, m \leqslant k / 2\end{cases}
$$

Доказательство. Нетрудно заметить, что $G(A) \in \mathscr{Q}(A)$. Таким образом, если взять $Q_{0}(A)=G(A)$, то выполняются условия теоремы 4 . 
Осталось найти супремумы значений норм строк и столбцов матриц $G(A)-L$. В данном случае нормы строк и столбцов совпадают и не зависят от $A$, поэтому

$$
b=d=\sqrt{m(1 / m-1 / k)^{2}+(k-m) 1 / k^{2}} .
$$

После арифметических преобразований в правой части получим, что

$$
b=d=\sqrt{(k-m) / m k} .
$$

Подставив это значение в оценку из теоремы 4, получим оценку, указанную в формулировке следствия.

\section{3. Оценка экспонента обобщенного кронекерова произведения матриц}

Определение 2. Пусть $A-$ квадратная $k \times k$ матрица, $\mathscr{B}=\left\{B_{i j}\right\}-$ упорядоченное множество из $k^{2}$ матриц размера $l \times l$. Назовем обобщенным кронекеровым произведением матрицы $A$ и множества матриц $\mathscr{B}$ матрицу $C=A \circ \mathscr{B}$ вида

$$
C=\left(\begin{array}{cccc}
a_{11} B_{11} & a_{12} B_{12} & \ldots & a_{1 k} B_{1 k} \\
a_{21} B_{21} & a_{22} B_{22} & \ldots & a_{2 k} B_{2 k} \\
& & \ldots & \\
a_{k 1} B_{k 1} & a_{k 2} B_{k 2} & \ldots & a_{k k} B_{k k}
\end{array}\right) .
$$

Частный случай обобщенного кронекерова произведения с матрицей $A$, все элементы которой равны 1 , использовался в [5].

Теорема 5. Пусть множество матрич $\mathscr{A}$ классно примитивно с экспонентом $\gamma(\mathscr{A})$, множество $\mathscr{D}$ классно примитивно с экспонентом $\gamma(\mathscr{D})$. Пусть

$$
\mathscr{C}=\{C=A \circ \mathscr{B} \mid A \in \mathscr{A}, \mathscr{B} \subseteq \mathscr{D}\}
$$

Тогда $\mathscr{C}$ классно примитивно и

$$
\gamma(C) \leqslant \max \{\gamma(\mathscr{A}), \gamma(\mathscr{D})\}
$$

Доказателъство. В матрице $C_{1} C_{2} \ldots C_{n}$ при $n \geqslant \max \{\gamma(\mathscr{A}), \gamma(\mathscr{D})\}$ рассмотрим блок размера $l \times l$. Пусть

$$
C_{m}=A_{m} \circ \mathscr{B}_{m}, \quad A_{m}=\left\|a_{i j}^{(m)}\right\| \in \mathscr{A}
$$

и матрица $B_{i j}^{(m)}$ находится на $i j$-м месте в упорядоченном множестве матриц $\mathscr{B}_{m}$. Положим

$$
D_{i j}=\sum_{i_{1}=1}^{k} \sum_{i_{2}=1}^{k} \ldots \sum_{i_{n-1}=1}^{k} a_{i i_{1}}^{(1)} a_{i_{1} i_{2}}^{(2)} \ldots a_{i_{n-1} j}^{(n)} B_{i i_{1}}^{(1)} B_{i_{1} i_{2}}^{(2)} \ldots B_{i_{n-1} j}^{(n)}
$$

Матрица $B_{i i_{1}}^{(1)} B_{i_{1} i_{2}}^{(2)} \ldots B_{i_{n-1} j}^{(n)}$ положительна, так как $n \geqslant \gamma(\mathscr{D})$. С другой стороны, существует набор $i_{1}, i_{2}, \ldots, i_{n-1}$ такой, что $a_{i i_{1}}^{(1)} a_{i_{1} i_{2}}^{(2)} \ldots a_{i_{n-1} j}^{(n)}>0$ поскольку $n \geqslant$ $\gamma(\mathscr{A})$. Следовательно, $D_{i j}>0$ при любых $i, j$. Отсюда и $C_{1} C_{2} \ldots C_{n}$ положительна, что и требовалось доказать. 
Эта теорема имеет применения в теории групп, а именно, для вычисления длины покрытия группы в заданной системе образующих.

Для дальнейшего изложения нам потребуются некоторые определения (все они взяты из [6]) и обозначения.

Пусть $G$ - произвольная конечная группа, $M-$ любое ее подмножество и $H=$ $\langle M\rangle$ - подгруппа группы $G$, порожденная множеством $M$. Так как $|H|<\infty$, каждый ее элемент $h$ представляется в виде произведения элементов из $M$. Минимальное число $l$ сомножителей среди всех таких представителей элемента $h$ назовем длиной элемента $h$ группы $H$ в системе образующих $M$ и обозначим $l(h ; M)$. Число

$$
l(H ; M)=\max _{h \in H} l(h, M)
$$

назовем длиной группы $H$ в системе образующих $M$.

Условимся подмножества из группы $G$ умножать как комплексы: $A B$ есть множество всех элементов вида $a b$, где $a \in A, b \in B$. Тогда системе образующих $M$ группы $H$ можно сопоставить последовательность подмножеств из $H: M, M^{2}, M^{3}, \ldots$ Множество $M^{i}$ назовем $i$-м слоем группы $H$ в системе образующих $M$.

Так как $|H|<\infty$, то найдется конечное число слоев $M^{i}$, объединение которых совпадает с $H$. Минимальное число таких слоев назовем шириной группы $H$ в системе образующих $M$ и обозначим $d(H ; M)$.

Нам потребуется ввести также определение квазидлины покрытия группы в множестве систем образующих.

Определение 3. Пусть $G$ - группа, $\mathscr{S}$ - некоторый набор подмножеств группы $G$. Множество $\mathscr{S}$ будем называть классно примитивным, если существует такое $n \in \mathbf{N}$, что для любой выборки с возвращением $S_{1}, S_{2}, \ldots, S_{n} \in \mathscr{S}$ имеет место равенство $S_{1} S_{2} \ldots S_{n}=G$.

Минимальное из таких чисел $n$ назовем квазидлиной покрытия группы $G$ в множестве систем образующих $\mathscr{S}$ и обозначим через $\widetilde{l}(G, \mathscr{S})$.

Будем обозначать $[G: H]$ - индекс подгруппы $H$ в группе $G$. Напомним, что если $H$ - нормальный делитель $G$, то $G$ можно представить как объединение непересекающихся смежных классов

$$
G=\bigcup_{m=1}^{[G: H]} g^{(m)} H
$$

Занумеруем все элементы конечной группы $G,|G|=k$, числами от 1 до $k$. Обозначим $A(S)=\left(a_{i j}\right)$ матрицу размера $k \times k$, где

$$
a_{i j}= \begin{cases}1, & \text { если } g_{i}^{-1} g_{j} \in S, \\ 0, & \text { если } g_{i}^{-1} g_{j} \notin S .\end{cases}
$$

Нетрудно заметить, что задача о квазидлине покрытия конечной группы $G$ в множестве систем образующих $\mathscr{S}$ сводится к подсчету экспонента множества матриц $\mathscr{A}=\{A(S) \mid S \in \mathscr{S}\}$. Поэтому из теоремы 5 получаем следующее утверждение.

Теорема 6. Пусть $G$ - конечная әруппа, $S$ - система образующих, $H$ - нормалъный делителъ $G$,

$$
G=\bigcup_{m=1}^{[G: H]} g^{(m)} H
$$


$L=G \mid H-\oint$ бактор-группа $G$ по $H$. Обозначим $S_{0}=\{g H\}$ те классы, в которые попали элементы из $S$,

$$
S_{i j}=H \cap S\left(g^{(j)}\right)^{-1} g^{(i)}, \quad i, j=1, \ldots,[G: H]
$$

и $\mathscr{S}$ - множество, состоящее из непустых подмножеств $S_{i j}$ группы $G$. ПТусть $d\left(L ; S_{0}\right)=1$. Тогда

$$
l(G ; S) \leqslant \max \left\{\tilde{l}\left(L ;\left\{S_{0}\right\}\right), \tilde{l}(H ; \mathscr{S})\right\} .
$$

Данная теорема позволяет свести задачу о длине покрытия группы, имеющей нормальный делитель, к. квазидлине покрытия фактор-группы и квазидлине покрытия нормального делителя в некотором множестве систем образующих.

\section{Список литературы}

1. Сачков В.Н., Ошкин И.Б., Экспоненты классов неотрицательных матриц. Дискретная математика (1993) 5, №2, 150-159.

2. Johnson C.R., Nylen P., Yamamoto's theorem for generalized singular values. Linear Algebra Appl. (1990) 128, 147-158.

3. Гантмахер Ф.Р., Теория матриц. Наука, Москва, 1988.

4. Беллман Р., Введение в теорию матриц. Наука, Москва, 1976.

5. Горчинский Ю.Н., Круглов И.А., Капитонов В.М., Вопросы теории распределений на конечных группах. Труды по дискретной математике (1997) 1, 85-112.

6. Глухов М.М., О числовых параметрах, связанных с заданием конечных групп системами образующих элементов. Труды по дискретной математике (1997) 1, 43-66.

Статья поступила 24.08.1998. 